\title{
Predictive Value of EGFR-PI3K-AKT-mTOR-Pathway Inhibitor Biomarkers for Head and Neck Squamous Cell Carcinoma: A Systematic Review
}

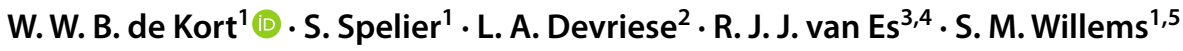

Accepted: 15 February 2021 / Published online: 8 March 2021

(c) The Author(s) 2021

\begin{abstract}
Background Understanding molecular pathogenesis of head and neck squamous cell carcinomas (HNSCC) has considerably improved in the last decades. As a result, novel therapeutic strategies have evolved, amongst which are epidermal growth factor receptor (EGFR)-targeted therapies. With the exception of cetuximab, targeted therapies for HNSCC have not yet been introduced into clinical practice. One important aspect of new treatment regimes in clinical practice is presence of robust biomarkers predictive for therapy response.

Methods We performed a systematic search in PubMed, Embase and the Cochrane library. Articles were included if they investigated a biomarker for targeted therapy in the EGFR-PI3K-AKT-mTOR-pathway.

Results Of 83 included articles, 52 were preclinical and 33 were clinical studies (two studies contained both a preclinical and a clinical part). We classified EGFR pathway inhibitor types and investigated the type of biomarker (biomarker on epigenetic, DNA, mRNA or protein level).

Conclusion Several EGFR-PI3K-AKT-mTOR-pathway inhibitor biomarkers have been researched for HNSCC but few of the investigated biomarkers have been adequately confirmed in clinical trials. A more systematic approach is needed to discover proper biomarkers as stratifying patients is essential to prevent unnecessary costs and side effects.
\end{abstract}

W. W. B. de Kort

W.W.B.dekort-3@umcutrecht.nl

S. Spelier

A.spelier-2@umcutrecht.nl

L. A. Devriese

L.A.Devriese@umcutrecht.nl

R. J. J. van Es

R.J.J.vanes@umcutrecht.nl

S. M. Willems

S.M.Willems@umcg.nl

1 Department of Pathology, University Medical Center Utrecht, PO Box 885500, 3508 GA Utrecht, The Netherlands

2 Department of Medical Oncology, University Medical Center Utrecht, PO Box 885500, 3508 GA Utrecht, The Netherlands

3 Department of Oral and Maxillofacial Surgery, University Medical Center Utrecht, PO Box 885500, 3508 GA Utrecht, The Netherlands

4 Department of Head and Neck Surgical Oncology, Utrecht Cancer Center, University Medical Center Utrecht, PO Box 885500, 3508 GA Utrecht, The Netherlands

5 Department of Pathology, University Medical Center Groningen, PO Box 30001, 9700 RB Groningen, The Netherlands

\section{Key Points}

Several EGFR-pathway inhibitor biomarkers have been researched for HNSCC but few of the investigated biomarkers have been adequately confirmed in clinical trials.

A systematic approach is needed to discover proper biomarkers as stratifying patients is essential to prevent unnecessary costs and side effects.

\section{Introduction}

Squamous cell carcinoma of the head and neck (HNSCC) is the sixth most common type of cancer, representing $6 \%$ of all cancer cases globally [1]. HNSCCs are challenging to treat, not only due to their anatomical location, which complicates surgery, but also due to their highly variable biological 
behaviour and treatment response. Robust factors predicting patient response to a specific treatment are lacking. HNSCC is primarily treated with surgery and (chemo)radiotherapy ((Ch)RT), or a combination of these modalities. Recently the application of immunotherapy, especially PDL1 inhibitors, was introduced $[2,3]$. Although some patients benefit from this treatment regime, relapse rates of over $50 \%$ are observed [4]. Moreover, treatment with chemo- and radiotherapy can result in significant morbidity and oropharyngeal discomfort such as mucositis and dermatitis, xerostomia, dysphagia, loss of taste, hoarseness, fibrosis, osteoradionecrosis, peripheral neuropathy, nephrotoxicity and ototoxicity, which affect the patient's quality of life [5, 6]. Once HNSCC recurs after surgery and (Ch)RT or if metastatic disease is present and immunotherapy appears ineffective, only limited palliative treatment options are available.

The understanding of the molecular pathogenesis of HNSCC has improved considerably in the last decade, resulting in the development of several targeted therapies for HNSCC [7]. In particular, therapies targeting the epidermal growth factor receptor (EGFR) have been explored extensively. For the clinician treating patients with HNSCC, knowledge concerning EGFR-inhibiting therapies is therefore relevant. EGFR is overexpressed in 50-90\% of HNSCC cases [8]. EGFR, also known as ErB1 or HER, is part of the ErbB family of cell surface receptors. ErbB signalling is involved in pivotal cellular processes including proliferation, anti-apoptotic signalling and differentiation via, among others, the phosphoinositide 3-kinase/protein kinase $\mathrm{B}$, also known as oncogene Thymoma in Ak-mouse/mammalian target of rapamycin (PI3K/AKT/mTOR) pathway, the most mutated pathway in HNSCC $[9,10]$. Overexpression of EGFR or proteins downstream the EGFR signalling pathway can drive malignant behaviour of a tumour [11, 12]. Several types of inhibitors have been described in the EGFRPI3K-AKT-mTOR pathway. Monoclonal antibodies (mABs) and tyrosine kinase inhibitors (TKIs) constitute the largest categories. PI3K-inhibitors and mTOR inhibitors make up a smaller group.

Currently, cetuximab is the only EGFR-targeting agent approved by the European Medicines Agency (EMA) for the treatment of HNSCC. Cetuximab can be administered concomitantly with radiotherapy in the curative setting to patients who have a contraindication for cisplatin. In recurrent and/or metastatic HNSCC, single-agent cetuximab can be administered as a palliative therapy. However, in the curative setting, two recent randomized controlled trials demonstrated that human papillomavirus (HPV)-positive patients with oropharyngeal carcinoma showed inferior overall survival if treated with cetuximab + RT as compared to cisplatin + RT $[13,14]$. This led to concerns about its efficacy or development of resistance. Another mAB is panitumumab, which like cetuximab binds the ectopic domain of EGFR [15].

Despite the development of many new targeting agents, both primary and acquired resistance to these agents has unfortunately resulted in low response rates when tested in patients. Additionally, albeit side effects of chemotherapy and radiotherapy are in general more severe, side effects of targeted therapy can still be serious [16-19]. For HNSCC, the only biomarker for a targeted therapy used in the clinic today is presence and severity of cetuximab-induced skin rash, which is predictive for response [20]. However, it is preferable that biomarkers can be used to predict effective patient response prior to treatment in order to circumvent unnecessary side effects and costs. We therefore solely focus on such biomarkers in this review. The aim of this study was to systematically review predictive biomarkers that are currently explored in HNSCC to predict the response of targeting therapies in the EGFR-PI3K-AKT-mTOR pathway. We also raise questions that need to be addressed in the future to guide patient treatment with EGFR inhibitors in the clinic.

\section{Materials and Methods}

A systematic search of PubMed, Embase database and the Cochrane library was performed on 25 January 2021. Articles were included if they were original articles and investigated a biomarker for targeted therapies interfering with the EGFR-PI3K-AKT-mTOR-pathway, in patients with any type of HNSCC. PI3K and mTOR inhibitors were included in this review as the PI3K-AKT-mTOR pathway harboured the highest percentage of mutations in HNSCC $[9,10]$. Reviews, commentaries and studies in a language other than English were excluded. This review is written in accordance with the PRISMA guidelines [21]. The search was set up in DDO-format (Domain, Determinant, Outcome). PubMed and Embase search syntaxes are displayed in Appendix 1 (Online Supplemental Material, OSM). The Cochrane library was searched with similar search terms. Two authors (WWBDK and SS) screened all articles on title and abstract. Conflicts were resolved by discussion. To prevent missing articles cross-reference screening was performed (Fig. 1). As various papers discussed several EGFR pathway inhibitors and reported preclinical and clinical data, the total number of studies is lower than the number of studies depicted in Fig. 2. Pre-clinical data is defined as data obtained using in vitro cell lines and xenograft models with both cell lines and patient-derived cells. The following data were extracted from the studies: study title, first author's name, date of publication, administered EGFR pathway inhibitor type, biomarker potentially correlated to response, analyzing techniques, sample size and additional treatment. Due to extensive variance in the studied biomarkers, data 
Fig. 1 Flowchart of search; date of search 25 January 2021. After screening 2700 abstracts, 223 papers were full-text screened. After adding crossreferences, a total number of 83 articles were included

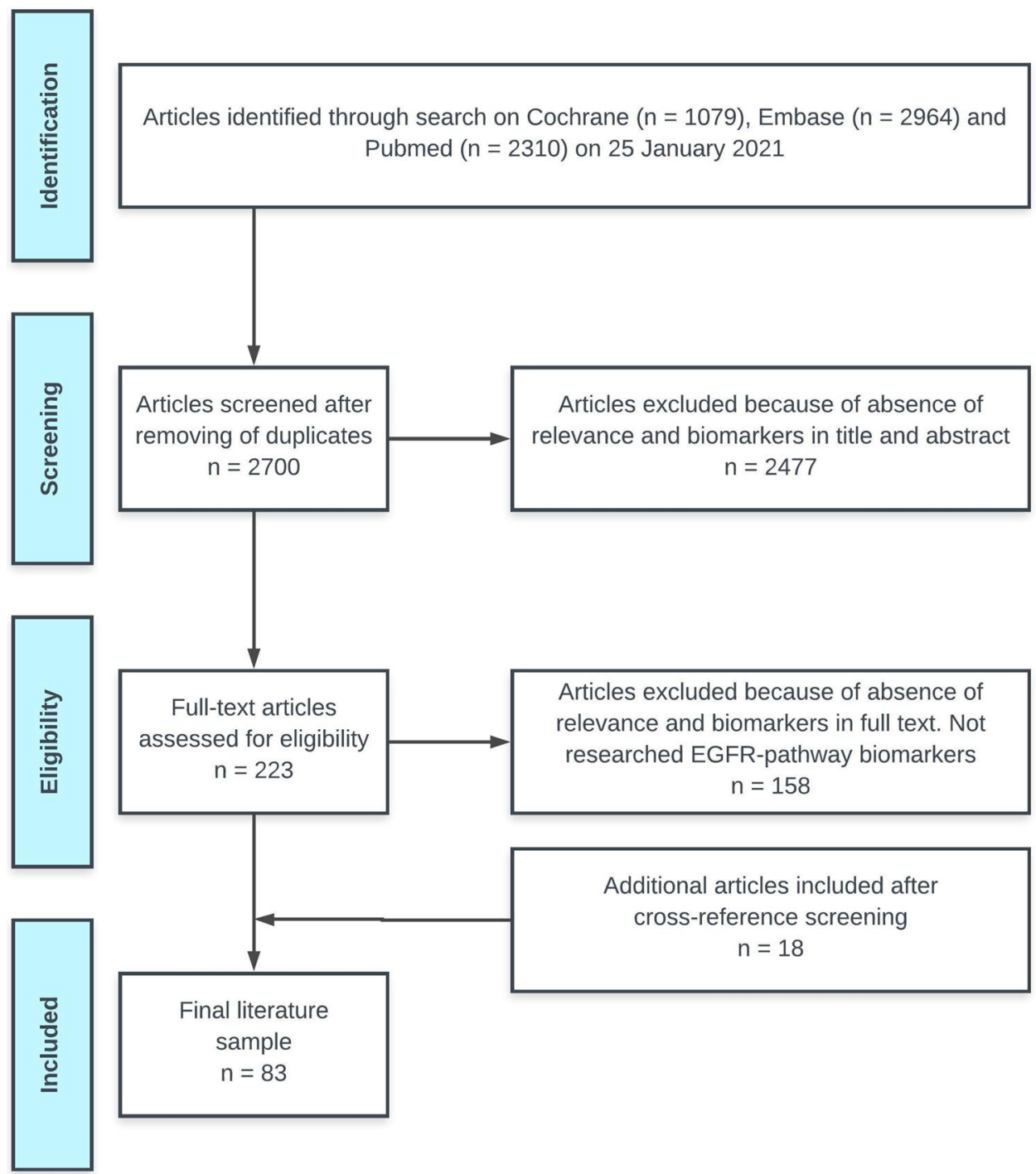

could not be quantitatively pooled and thus a meta-analysis was not performed.

\section{Results}

Of the 83 included articles, 52 articles were preclinical and discussed in vitro experiments and 33 articles discussed the results of clinical trials performed in patients (Fig. 1). Some studies were placed in both categories. Fifteen clinical studies were cohort studies, five phase II studies, one phase II + phase III, and five phase III studies. Within these two subgroups distinctions are made between (1) the different EGFR pathway inhibitors and (2) the type of biomarker (biomarker on epigenetic, DNA, mRNA or protein level). A schematic overview of the EGFR pathway with numbers of included studies per agent is shown in Fig. 2. The number of biomarkers researched per EGFR pathway inhibitor and biomarker-level are displayed in Table 1. Note that one biomarker researched by two studies is counted as two researched biomarkers in Table 1. For a detailed description of all biomarkers, we refer to OSM Table 1a-d (preclinical) and OSM Table 2a-d (clinical).

\subsection{Biomarkers Predicting Response to mABs (OSM Tables $1 A$ and $2 A$ )}

Cetuximab and panitumumab bind the extracellular domain of EGFR with higher affinity than natural ligands, thus inhibiting EGFR activation [22]. Cetuximab additionally stimulates internalization of EGFR [23]. Moreover, being an immunoglobulin $\mathrm{G}$ molecule, cetuximab stimulates antibody-dependent cell cytotoxicity [24, 25]. 
Fig. 2 Simplified overview of EGFR pathway, with numbers of studies included per EGFR pathway inhibitor target. Important note: Several studies investigated more than one inhibitor; the total number of studies depicted in this figure exceeds the total number of inclusions. EGFR can be bound by natural ligands such as EGF, leading to dimerization of two EGFR monomers and subsequent activation of the intracellular tyrosine kinase domain of EGFR. This results in activation of the downstream signalling cascade via PI3K/AKT/mTOR or KRAS/BRAF/MEK/ERK, signalling resulting in cellular processes such as cell proliferation or differentiation. Illustration made with BioRender ${ }^{\circledR}$

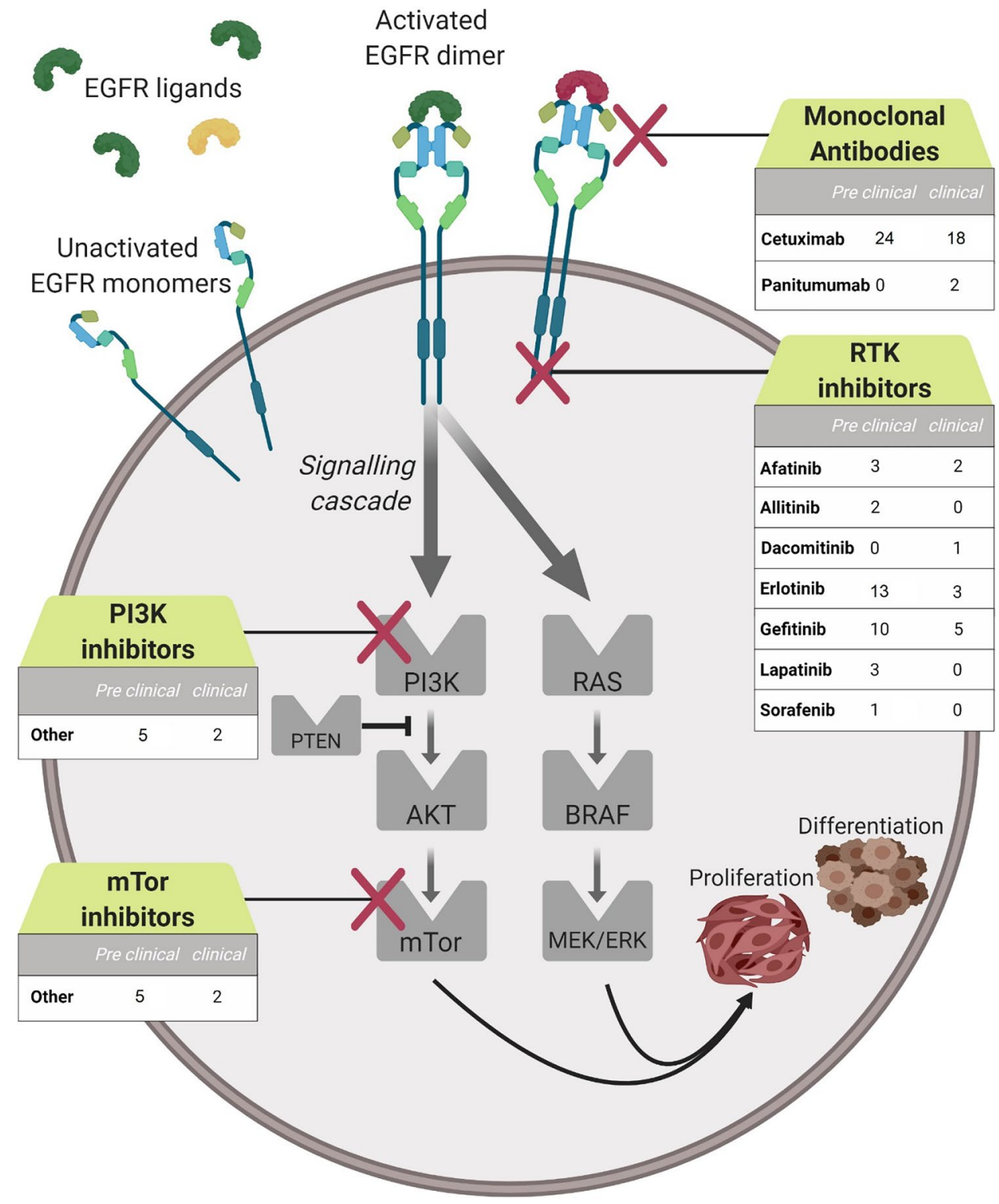

\subsubsection{EGFR Overexpression (Cetuximab)}

It has been hypothesized that EGFR overexpression correlates with response to cetuximab. The increase in expression of EGFR molecules could result in increased binding of cetuximab resulting in a stronger inhibitory effect. Six studies showed a positive correlation between EGFR overexpression and cetuximab response [26-31]. Other studies obtained conflicting results, one study mentioned that about half of the preclinical tumour models did benefit from cetuximab while lacking EGFR amplification [26] and two studies were based on only two and three cell lines, respectively [27, 30]. Moreover, the fact that five clinical studies [32-37] and six preclinical studies [28, 29, 38-41] failed to show any correlation between EGFR overexpression and cetuximab response implies that EGFR expression is not suitable as a single biomarker.

\subsubsection{ErbB Family Member Overexpression and Activation (Cetuximab)}

The ErbB family of proteins contains four RTKs, Her1 (EGFR, ErbB1), Her2 (Neu, ErbB2), Her3 (ErbB3) and HER4 (erbB4). Apart from HER1 (EGFR), it has been hypothesized that the expression/activation-level HER2, HER3 and HER4 correlates with cetuximab response. Preclinical studies showed that HER3 expression [40] and phosphorylated HER2/HER3 [42] correlated negatively with cetuximab response. In one preclinical study, expression levels of HER4 on tumour were found to correlate positively 
Table 1 Numbers of biomarkers researched per EGFR pathway inhibitor type and per biomarker-level

\begin{tabular}{|c|c|c|c|c|c|c|c|c|c|c|}
\hline & \multicolumn{10}{|c|}{ Biomarker level } \\
\hline & \multicolumn{2}{|l|}{ Epigenetic } & \multicolumn{2}{|l|}{ DNA } & \multicolumn{2}{|l|}{ RNA } & \multicolumn{2}{|l|}{ Protein } & \multicolumn{2}{|l|}{ Other } \\
\hline & Pre-clinical & Clinical & Pre-clinical & Clinical & Pre-clinical & Clinical & Pre-clinical & Clinical & Pre-clinical & Clinical \\
\hline \multicolumn{11}{|c|}{ Monoclonal antibodies } \\
\hline Cetuximab & 2 & - & 8 & 9 & 11 & 12 & 31 & 15 & 1 & - \\
\hline Panitumumab & - & - & - & - & - & 4 & - & 1 & - & - \\
\hline \multicolumn{11}{|l|}{ RTK inhibitors } \\
\hline Gefitinib & - & - & 5 & 3 & 2 & 1 & 22 & 4 & - & - \\
\hline Erlotinib & - & - & 7 & 5 & 1 & 1 & 6 & 2 & 2 & - \\
\hline Afatinib & - & - & 2 & 1 & - & 1 & 2 & 8 & - & - \\
\hline Lapatinib & - & - & 1 & - & - & - & 2 & - & - & - \\
\hline Allitinib & - & - & 2 & - & - & - & - & - & - & - \\
\hline Sorafenib & - & - & - & - & - & - & 1 & - & - & - \\
\hline Dacomitinib & - & - & - & 1 & - & - & - & - & - & - \\
\hline \multicolumn{11}{|l|}{ PI3K inhibitors } \\
\hline & - & - & 12 & 2 & - & - & - & 1 & - & 2 \\
\hline \multicolumn{11}{|l|}{ mTOR inhibitors } \\
\hline & - & - & 2 & 1 & 5 & - & 3 & - & 2 & 1 \\
\hline
\end{tabular}

Important note: All the studies were added separately. For example: EGRF expression investigated for gefitinib response in three studies is displayed in the table as 3 , not as 1 . Several studies researched multiple biomarkers; because of this the total number of biomarkers exceeds the number of included studies

with patient response to cetuximab [30]. However, the authors investigated only two cell lines. Two other preclinical studies reported no correlation between expression or activation of ErbB family members and cetuximab response [28, 38]. Clinical studies in HNSCC elaborating on these preclinical results are lacking. Investigating the potential of the ErbB family members as biomarkers for HNSCC in clinical trials is recommended.

\subsubsection{EGFR Ligand Overexpression (Cetuximab)}

Apart by EGF, EGFR can be activated by other EGFRligands like heparin-binding EGF (HB-EGF), transforming growth factor- $\alpha$ (TGF- $\alpha$ ), epiregulin (EREG), and amphiregulin (AREG). Expression of the EGFR ligands AREG [43] and EREG [39, 43] correlated positively with cetuximab response. Interestingly, mRNA levels of yes-associatedprotein 1 (YAP1) correlated negatively with cetuximab response [44]. YAP1 is an activating transcription factor of among others AREG, explaining an indirect negative correlation. However, YAP1 also is a transcriptional co-activator for several genes involved in anti-apoptosis and proliferation, resulting in a potential bias when drawing conclusion about the correlation between AREG and cetuximab response per se. Ansell et al. could not correlate EREG and AREG expression to cetuximab response in a preclinical study on tongue cancer [45]. In clinical studies, contradicting results are demonstrated as well: Kogashiwa et al. described a positive correlation between EREG and AREG mRNA expression and cetuximab response [46], whereas AREG protein levels correlated negatively with cetuximab response in the study of Tinhofer et al. [35] Tinhofer et al. base their conclusions on immuno-histochemistry, which is more conclusive than RNA levels determined on quantitative polymerase chain reaction (qPCR) [46].

Three preclinical studies investigated EGFR-ligands HBEGF [47, 48] and EGF [45] as biomarkers for cetuximab response. Ligand expression correlated negatively with cetuximab response in all three studies. No clinical studies elaborate on these findings. EGFR ligands seem potential biomarkers for cetuximab response in HNSCC patients, although contradictory results warrant further clinical studies using larger cohorts of patients.

\subsubsection{Epithelial to Mesenchymal Transition (Cetuximab)}

Epithelial to mesenchymal transition (EMT) is a process pivotal in (early) developmental processes as well as for metastasis of tumour cells. EMT is characterized by loss of epithelial cell characteristics like E-cadherin expression and gain of mesenchymal characteristics like vimentin and fibronectin expression. EMT can result in cell detachment from adjacent epithelial cells and subsequent cell migration [49]. E-cadherin/vimentin expression did not correlate with cetuximab response in one preclinical study [40]. Four preclinical studies reported a positive correlation between 
cetuximab response and the absence/presence of EMT-like features. Expression of epithelial markers as keratin 13/14 (KRT13/14) [48] and possession of a basal epithelial gene expression signature [50] correlated positively with cetuximab response. Accordingly, expression of EMT markers correlated negatively with cetuximab response in HNSCC cell lines [48].

Keck et al. discovered five subtypes of HNSCC using gene expression-based consensus clustering, copy number profiling and HPV status [51]. The 'basal' subtype was characterized as HPV negative with strong hypoxic differentiation (enrichment for hypoxia signalling) and prominent EGFR/HER signalling. HNSCC patients with this basal expression profile were more responsive to cetuximab [52]. On the contrary, expression of matrix metalloproteinase 9 (MMP9), known to be expressed during EMT [53], correlated positively with cetuximab response in a clinical study [32]. This correlation is supported by the fact that MMPs can liberate EGFR ligands from the extracellular matrix, thereby potentially promoting EGFR signalling leading to tumour cells becoming extra sensitive to EGFR inhibition [32]. This correlation therefore does not immediately interfere with the previous discussed results on EMT as a predictive biomarker for $\mathrm{mAB}$ resistance.

To summarize, it is hard to draw conclusions due to the limited number of clinical inclusions; yet we see the potential of EMT markers like e-cadherin expression to serve as potential biomarkers of response to EGFR mABs.

\subsubsection{Other Biomarkers (Cetuximab)}

Several biomarkers were investigated in only a small number of studies. The rapid development of sequencing technologies and microarrays enables high-throughput genomic expression or proteomic profiling of tumour material. This has led to the introduction of gene signatures: specific expression profiles correlated to a cancer subtype, or even to therapy response [54]. MammaPrint ${ }^{\circledR}$ (Agendia, The Netherlands) highlights the potential of such expression profiles. By investigating the expression of 70 genes it aids in stratification of breast cancer patients into low- and high-risk groups, advising on treatment strategies [55]. In HNSCC cell lines, a specific RNA expression profile correlated with an increased response to cetuximab + RT26. Similarly, a specific miRNA expression profile correlated positively to cetuximab $+\mathrm{CT}$ response in a clinical trial [56]. The potential of expression profiles as biomarkers is not very surprising, as a profile in fact is a combination of multiple biomarkers. Therefore, studies on expression profiles as biomarkers deserve more confirmation in prognostic clinical trials.

Various other preclinical studies describe biomarkers that correlate negatively with cetuximab response, like possession of EGFR-K single-nucleotide polymorphism on DNA level [57], which was validated in patients [58]. Two preclinical studies demonstrated that growth factor receptor (c)MET expression correlated negatively with cetuximab response [40, 42], whereas two clinical studies could not verify this correlation $[32,59]$. Several additional biomarkers correlating to cetuximab resistance were identified: mutations downstream the EGFR signalling cascade (PI3KCA, KRAS, HRAS, BRAF) [37, 41], chemokine ligand 14(CXCL14) expression [60], tyrosine kinase receptor AXL expression [61], fibroblast growth (FGF) receptor 3 expression [43] and pAKT expression [29, 62].

Four biomarkers were described in clinical studies that correlate positively with cetuximab response: (1) the possession of a germline mutation in a microRNA-binding site in KRAS [63], (2) phosphatase and tensin homolog (PTEN) protein expression $[36,59,64]$ supported by a study where PTEN knock down correlates with cetuximab resistance [65], although no correlation was found in two studies [37, 66]; (3) VEGF protein/Interleukin(IL)-6 expression [67]; and (4) expression of IL-1 ligands [68]. Four clinical studies describe biomarkers that correlate negatively with cetuximab response: (1) KRAS/HRAS mutations [57], (2) presence of the EGFR-variant EGFRvIII [35], (3) phosphorylated AKT levels [69] and (4) the presence of a long noncoding RNA transcript fusion [70].

KRAS mutations occur in $<5 \%$ of HNSCC cases [71]. Braig et al. [57, 58] underline the potential of this biomarker, albeit its relevance for a relatively small subgroup of the HNSCC patients. H-RAS mutations occur slightly more frequently than KRAS mutations (11\%) [72]. Few studies describe the role of KRAS or HRAS mutations in HNSCC treatment with EGFR inhibitors and prospective clinical studies are lacking.

\subsubsection{Biomarkers for Panitumumab}

Panitumumab, like cetuximab, binds the ectopic domain of EGFR [15]. In this review, no studies were included on the relation between RAS status and panitumumab response in HNSCC patients. Two clinical studies investigated prognostic biomarkers for panitumumab response. Expression of Cluster3 gene signature (a hypoxia signature with miRNAgene expression of ARRDC4, CRCT1, IL36G, KLK10 and PLA2G4E) or the individual expression of seven different miRNAs [15] and a negative P16 status [73] correlate with improved panitumumab response in clinical trials. It is impossible to draw conclusions because studies of biomarkers for panitumumab are limited. 


\subsection{Receptor Tyrosine Kinase (RTK) Inhibitors}

EGFR-targeting TKIs are small molecules that cross the cell membrane and intracellularly inhibit the receptor tyrosine kinase (RTK) of EGFR by binding and blocking the adenosine triphosphate(ATP) binding site, thereby inhibiting downstream EGFR phosphorylation essential for downstream signaling [74]. Downstream of EGFR, HNSCCs often show oncogenic alterations in the PI3K/AKT/mTOR pathway.

\subsubsection{Biomarkers for Gefitinib}

Gefitinib is a TKI targeting EGFR [28]. For gefitinib, seven biomarkers have been identified that successfully predict patient response. EGFR amplification correlated with gefitinib response in three preclinical studies $[28,75,76]$. This positive correlation between EGFR amplification and gefitinib response was not found in two other preclinical [77, $78]$ and two clinical studies $[79,80]$. As for cetuximab, the results are heterogeneous and further studies are warranted.

Several other biomarkers that correlated with response to gefitinib were: a specific RNA signature [75], MET/AREG expression [76], ANO1 (a calcium-activated chloride channel that interacts with EGFR) expression [81] and protein kinase C-e (PKCe) expression [82]. Two clinical studies described biomarkers on protein level that correlated positively with response to gefitinib: insulin-like growth factor (IGF) 1 receptor expression [83] and MMP11 expression [79].

Eleven biomarkers correlated negatively with gefitinib response in the preclinical studies, all on protein level; HER3 and phosphorylated HER2 expression [28], expression of AREG/hepatocyte growth factor(HGF)/IGF1/FGF1/ FGF2/IGFR/EGFR/MET [78], EMT-associated protein expression alterations (increased vimentin and decreased e-cadherin) [84], cortactin protein levels [85] and a protein signature (granulocyte-macrophage colony-stimulating factor (GM-CSF), IL-8, metallopeptidase inhibitor-1 (TIMP-1), VEGF) [86]. One clinical study reports that the possession of a stable EGFR-AS1 long noncoding RNA variant is associated with gefitinib resistance [87].

Biomarkers in two clinical studies did not correlate to gefitinib response: The protein signature as described above (GM-CSF, IL-8, TIMP-1, VEGF) [86] and EGFR amplification/EGFR kinase-domain mutations [80].

\subsubsection{Biomarkers for Erlotinib}

Erlotinib is a TKI targeting EGFR. Five preclinical studies showed a positive correlation of biomarkers with erlotinib response. ANO1 protein expression [81], pEGFR expression [31], presence of mitogen-activated protein kinase
(MAPK)1p.D321N mutation [88] or MAPK1 E322K mutation [89] and EREG expression [90]. Also preclinically, six biomarkers correlated negatively with erlotinib response, three of which can be linked to EMT characteristics similar to those described for cetuximab; SNAIL (an e-cadherin inhibitor) overexpression correlates negatively with erlotinib response [91]; a combination of low EGFR and negative e-cadherin protein expression correlates negatively with erlotinib response [92]; EMT characteristics demonstrated in a functional assay correlate negatively with erlotinib response [93]. Although these results are promising, the influence of EMT-related biomarkers on erlotinib response is not validated in clinical trials.

Three other biomarkers correlated negatively with erlotinib response; PTEN knockout [65], HMG-box transcription factor 1 (HBP1) knockout [94], and a protein signature of GM-CSF, IL-8, TIMP-1 and VEGF [86]. However, this signature could not be correlated to patient resistance clinically due to small cohort size.

In the clinical studies, none of the biomarkers correlated significantly with erlotinib response [80, 95, 96].

\subsubsection{Biomarkers for Afatinib}

Afatinib is a TKI targeting both EGFR, HER2 and HER4 [97]. In preclinical studies, AKT expression [98] and EGFR amplification [99] correlated positively with afatinib response and one clinical trial confirmed that EGFR amplification correlated positively with afatinib response [100].

A specific protein signature correlated negatively with afatinib response in a preclinical study [86].

Possession of a tumour protein p53 (TP53) wildtype and a specific cluster 3 hypoxia profile correlated positively with afatinib response [97]. In another clinical study, PTEN expression on protein level correlated positively with afatinib response [100]. Contradictorily, two clinical studies elaborated on the absence of a correlation between PTEN expression and afatinib response [97, 99]. Machiels et al., however, emphasize that the number of patients in their study was quite low (30) and that the clinical background of their treated patients was different (palliative vs. curative intent) [97].

\subsubsection{Biomarkers for Lapatinib/Allitinib/Sorafenib/ Dacomitinib}

Lapatinib and allitinib are dual kinase inhibitors targeting both EGFR and HER2 [98, 101], sorafenib is a multi-kinase inhibitor also targeting EGFR [102] and dacomitinib, a panHER inhibitor [103].

Lapatinib, allitinib and sorafenib were only studied in preclinical studies. On a DNA level, a specific gene expression profile of nine distinct genetic loci including gains of HER2 
and EGFR and loss of CDKN2A correlated positively with lapatinib response [101]. On a protein level ANO1 expression correlated positively lapatinib response [81], while PKCe expression correlated with worse response [82]. AKT expression correlated positively with allitinib response [98]. In contrast, KRAS mutations correlated negatively with allitinib response [104].

MET expression was investigated as a biomarker for sorafenib, but did not correlate with response [102].

A clinical trial on dacomitinib showed that protein reversionless 3-like (REV3L) mutations correlated positively with dacomitinib response [103].

\subsection{PI3K Inhibitors}

PI3Ks are a family of related intracellular signal transducer enzymes that include the oncogene PIK3CA and tumour suppressor gene PTEN. PI3K-inhibitors inhibit one or more signal transducer enzymes. HNSCCs are known to contain activating mutations in PIK3CA $[9,10]$. As a result, PI3Ktargeting agents resulting in PI $3 \mathrm{~K}$ inhibition and subsequent tumour suppression have consequently gained interest as emerging therapeutics for HNSCC [105]. Constitutive activation of the PI3K pathway has been described as a potential of resistance to EGFR inhibitors. Therefore, therapeutic agents targeting the PI3K/AKT/mTOR pathway have become an important focus in HNSCC research [41, 62].

\subsubsection{Biomarkers for PI3K Inhibitors}

Based on in vitro studies, cancers carrying PIK3CA mutations are thought to be more responsive to specific $\mathrm{PI} 3 \mathrm{~K} \alpha$ inhibitors [105]. Presence of oncogene PI3KCA mutations correlated with improved response to PI3K-inhibitors in three preclinical studies [77, 106, 107] and in the preclinical and clinical part of the study by Lui et al. [9] Two other preclinical studies could not confirm this correlation [105, 108].

Also in preclinical studies, the presence of Notch1 mutations [107], PI3KCA amplification [77], EGFR/AKT1 amplification and CUB and Sushi multiple domains 1 (CSMD1) deletion [105] correlated with improved response. However, PIK3CA amplification did not correlate with response in another preclinical study [106].

In a clinical study, HPV-negative tumour status, the presence of TP53 mutations and an increase in tumour infiltration of CD8+ T-cells correlated with improved response to buparlisib, a PIK3CA inhibitor [109]. Because the number of studies investigating biomarkers for Pi3K inhibitors is low, drawing firm conclusions on clinical implementation is not yet possible.

\section{4 mTOR Inhibitors}

Additional to the development of PI3K inhibitors, inhibitors of mTOR have been explored to treat HNSCC [106]. mTOR inhibitors inhibit the mechanistic target of rapamycin (mTOR).

\subsubsection{Biomarkers for mTOR Inhibitors}

For mTOR inhibitors, similar biomarkers as for PI3K inhibitors have been suggested to be relevant. Presence of PI3KCA mutations correlated with improved response to mTOR inhibitors in three preclinical studies [106, 107, 110], like the presence of Notch1 mutations in one study [107]. PI3KCA amplification, loss of PTEN and TP53 mutations did not correlate with improved response to mTOR inhibitors [106, 111]. Niehr et al. describe improved mTOR inhibitor response in patients with cisplatin resistance, and worse mTOR inhibitor response with EGFR protein expression and cetuximab resistance [111]. Response to the mTORinhibitor temsirolimus did not correlate with mRNA expression of mTOR pathway members Akt1, mTOR, RPS6KB1, FKBP1B and TSC1 in another preclinical study [112].

Clinically, baseline caspase-3 activity correlated negatively with temsirolimus response in one study [113]. Also PI3KCA mutations and HPV status did not correlate to mTOR response [114].

Due to the limited number of clinical inclusions, it is impossible to draw conclusions about predictive biomarkers with regard to response to mTOR inhibitors.

\section{Discussion}

The majority of HNSCC tumours depend on EGFR pathway activation for processes like cell proliferation, anti-apoptotic signalling, angiogenesis and even metastasis. Consequently, EGFR pathway inhibitors have received attention as potential therapeutic agents. However, primary and acquired resistance to EGFR targeting agents often occurs, resulting in low response rates and tumour recurrence. There are many mechanisms of resistance to EGFR targeting agents. Tumour cells resistant to cetuximab can re-activate pro-angiogenic factors via alternative pathways, increasing VEGF leading to neovascularisation [115]. Cetuximab treatment can induce EGFR internalization and degradation and HER-family members can be upregulated to bypass EGFR blockade by cetuximab [116]. For other TKIs, activation of alternative pathways also leads to resistance [117]. This underscores the need for biomarkers predictive for response to EGFR pathway inhibitors prior to treatment. The only biomarker used at present is not molecular but clinical, i.e. the presence and severity of skin rash, which is predictive for cetuximab 
response [20]. Apart from the EGFR-PI3K-AKT-mTOR pathway, the immune environment of the tumour is also interesting regarding targeted therapy response [118]. Here biomarkers for immunotherapy were not included, although immunotherapy in HNSCC might be promising. In this review studies investigating a range of biomarkers for the response to different EGFR-targeting agents have shown conflicting results. This review aimed to give an overview and analysis of these studies published up to 25 January 2021.

We encountered several hurdles that impaired us from drawing firm conclusions on the applicability of the biomarkers included in this review. First, the number of clinical studies investigating biomarkers in general is low, with cetuximab being an exception. Second, many of the studies contained a very small sample size, which makes it hard to draw conclusions about the effect of the correlations found. Most biomarkers have been investigated in only a limited number of studies and subsequently have not been followed up by clinical trials, thus confirmation of their relevance is lacking. Exceptions are EGFR amplification and biomarkers relating to EMT. Also, the discrepancies between exact treatments in clinical trials as well as the differences in the clinical background in patients (recurrent vs. metastatic, or palliative vs. curative) limit the possibility to draw firm conclusions. Moreover, head and neck cancers make up a heterogenic group of tumours both HPV-positive and -negative in different sub-sites of the oral cavity, pharynx and larynx.

Albeit such differences are hard to avoid, it is challenging to compare clinical studies. Concerning additional treatments, all the discussed targeted therapies were given concurrently with a form of traditional chemotherapy and/or radiotherapy, which limits drawing conclusions about the predictive value of that biomarker for the EGFR pathway inhibitor of interest. All these limitations are applicable for the whole predictive biomarker field. We therefore underline the need for a more systematic approach to tackle these problems. This could be by adding two mandatory components in clinical trials: (1) the assessment of potential biomarkers for the specific EGFR pathway inhibitor studies in that clinical trial, by preferably an independent expert (panel) prior to submitting a proposal for a clinical trial, and (2) including these potential biomarker assays in the clinical trial. On the other hand, it should be questioned whether the classic approach via clinical trials should be continued. Clearly the development of biomarkers through clinical trials has not resulted in any relevant biomarker. As every tumour is genetically unique it is hard to set up a decent biomarker trial with high numbers of comparable tumours. Biankin et al. therefore advocate other types of studies in personalised medicine towards more patient-centred trials [119].

Although this would require a big effort in the field, this review underlines the need for increased attention to biomarkers for targeted therapies. Patients themselves will benefit from pre-treatment stratification as this will prevent unnecessary side effects in non-responders. Also, despite the fact that screening all patients before treatment is expensive, we believe that the advantages will outweigh time and costs. For example, Cetuximab costs approximately $€ 8000$ per HNSCC patient, excluding hospital administration and follow-up. For screening every patient targeted next generation sequencing (NGS) is most commonly used. Targeted NGS panels are panels of approximately 70 cancer-related genes that are read using targeted NGS. Using NGS, point mutations and small indels can be detected, although larger, more complex chromosomal alterations (including large deletions, gene fusions or large CNV) cannot be detected using this approach. On average, performing NGS for one patient costs approximately $€ 600$ (including material and analysis). Screening 13 patients with NGS is as expensive as treating one patient with cetuximab. Screening techniques will become cheaper resulting in more patients who can be screened for the cost of one cetuximab treatment. With a reliable biomarker all patients will be screened and only the patients in whom the drug is effective will be treated, which in the end is cheaper.

This review emphasizes the lack of clinically validated biomarkers with high predictive value. EGFR expression is studied in many papers, but the results are too heterogeneous to consider any of the proposed biomarkers suitable for clinical response prediction in general. We think the application of a single biomarker is not adequate in predicting patient response. Studies integrating expression or mutation status of multiple genes and/or proteins have more potential to predict therapy response as they contain a combination of single biomarkers. A DNA/RNA/protein profile is a barcode of many biomarkers combined. In this review several papers found the predictive value of such profiles on DNA [48, 101], RNA [15, 26, 50, 52, 56, 75, 97] and protein level $[84,86]$. Based on these papers, as well as promising advances in other similar fields (as with the previously mentioned example of the MammaPrint), DNA/RNA/ protein profiles deserve further validation as biomarkers for EGFR-targeted therapies for HNSCC patients. In the future, sequencing will become cheaper and will characterise more expression profiles. Although such an expression profile for the prediction of nodal metastasis in HNSCC was created previously, its applicability in daily clinical practice has not yet been successful [120].

We additionally want to highlight a specific type of biomarker that is relatively new in the personalized medicine field-patient-derived organoids. Organoids are threedimensional structures that are adult stem-cell derived, and to some degree resemble the tissue or tumour they are derived of. Organoids derived from tumours from patients recapitulate the original tumours in tissues such as the colon, 
small intestine, pancreas and prostate [121-123]. This enables correlating in vitro organoid drug response to the response of patients in the clinic, as shown in a clinical study for organoids derived from gastrointestinal cancer [124]. Recently, HNSCC-derived organoids have been established and several targeted therapies were tested on patient-derived HNSCC organoids [41]. Differences between HNSCC organoid lines in response to cetuximab have been demonstrated, and initial comparisons to clinical data of the corresponding patients showed the potential of organoid technology in the predictive biomarker field.

In conclusion, several EGFR-pathway inhibitor biomarkers have been researched for HNSCC but few of the investigated biomarkers have been adequately confirmed in clinical trials. A more systematic approach is needed to discover proper biomarkers as stratifying patients is essential to prevent unnecessary costs and side effects.

Supplementary Information The online version contains supplementary material available at https://doi.org/10.1007/s40291-021-00518-6.

\section{Declarations}

Funding This research did not receive any specific grant from funding agencies in the public, commercial or not-for-profit sectors.

Conflict of interest The authors declare no potential conflicts of interest.

Ethics approval Not applicable.

Consent to participate Not applicable.

Data availability Not applicable.

Consent for publication Not applicable.

Code availability Not applicable.

Open Access This article is licensed under a Creative Commons Attribution-NonCommercial 4.0 International License, which permits any non-commercial use, sharing, adaptation, distribution and reproduction in any medium or format, as long as you give appropriate credit to the original author(s) and the source, provide a link to the Creative Commons licence, and indicate if changes were made. The images or other third party material in this article are included in the article's Creative Commons licence, unless indicated otherwise in a credit line to the material. If material is not included in the article's Creative Commons licence and your intended use is not permitted by statutory regulation or exceeds the permitted use, you will need to obtain permission directly from the copyright holder. To view a copy of this licence, visit http://creativecommons.org/licenses/by-nc/4.0/.

\section{References}

1. Fitzmaurice C, Allen C, Barber RM, et al. Global, regional, and national cancer incidence, mortality, years of life lost, years lived with disability, and disability-adjusted life-years for 32 cancer groups, 1990 to 2015: a systematic analysis for the global burden of disease study global burden of disease cancer collaboration. JAMA Oncol. 2017;3(4):524-48. https://doi.org/10.1001/jamao ncol.2016.5688.

2. Cramer JD, Burtness B, Le QT, Ferris RL. The changing therapeutic landscape of head and neck cancer. Nat Rev Clin Oncol. 2019;16(11):669-83. https://doi.org/10.1038/s4157 1-019-0227-z.

3. Chow LQM. Head and neck cancer. N Engl J Med. 2020;382(1):60-72. https://doi.org/10.1056/NEJMra1715715.

4. Argiris, AthanassiosView Profile; Karamouzis, Michalis V; Raben, DavidView Profile; Ferris RL. Head and neck cancerProQuest. 2008. https://doi.org/10.1016/S0140-6736(08)60728 $-\mathrm{X}$.

5. Trotti A, Bellm LA, Epstein JB, et al. Mucositis incidence, severity and associated outcomes in patients with head and neck cancer receiving radiotherapy with or without chemotherapy: a systematic literature review. Radiother Oncol. 2003;66(3):253-62. https://doi.org/10.1016/S0167-8140(02)00404-8.

6. Rosenthal DI, Lewin JS, Eisbruch A. Prevention and treatment of dysphagia and aspiration after chemoradiation for head and neck cancer. J Clin Oncol. 2006;24(17):2636-43. https://doi. org/10.1200/JCO.2006.06.0079.

7. Gougis P, Moreau Bachelard C, Kamal M, et al. Clinical development of molecular targeted therapy in head and neck squamous cell carcinoma. JNCI Cancer Spectr. 2019;3(4):1-12. https://doi. org/10.1093/jncics/pkz055.

8. Bossi P, Resteghini C, Paielli N, Licitra L, Pilotti S, Perrone F. Prognostic and predictive value of EGFR in head and neck squamous cell carcinoma. Oncotarget. 2016;7(45):74362-79. https:// doi.org/10.18632/oncotarget.11413.

9. Lui VWY, Hedberg ML, Li H, et al. Frequent mutation of the PI3K pathway in head and neck cancer defines predictive biomarkers. Cancer Discov. 2013;3(7):761-9. https://doi. org/10.1158/2159-8290.CD-13-0103.

10. Iglesias-Bartolome R, Martin D, Silvio GJ. Exploiting the head and neck cancer oncogenome: widespread PI3K-mTOR pathway alterations and novel molecular targets. Cancer Discov. 2013;3(7):722-5. https://doi.org/10.1158/2159-8290. CD-13-0239.

11. Normanno N, De Luca A, Bianco C, et al. Epidermal growth factor receptor (EGFR) signaling in cancer. Gene. 2006;366(1):216. https://doi.org/10.1016/j.gene.2005.10.018.

12. Glazer CA, Chang SS, Ha PK, Califano JA. Applying the molecular biology and epigenetics of head and neck cancer in everyday clinical practice. Oral Oncol. 2009;45(4-5):440-6. https://doi. org/10.1016/j.oraloncology.2008.05.013.

13. Mehanna H, Robinson M, Hartley A, et al. Radiotherapy plus cisplatin or cetuximab in low-risk human papillomavirus-positive oropharyngeal cancer (De-ESCALaTE HPV): an open-label randomised controlled phase 3 trial. Lancet. 2019;393(10166):5160. https://doi.org/10.1016/S0140-6736(18)32752-1.

14. Gillison ML, Trotti AM, Harris J, et al. Radiotherapy plus cetuximab or cisplatin in human papillomavirus-positive oropharyngeal cancer (NRG Oncology RTOG 1016): a randomised, multicentre, non-inferiority trial. Lancet. 2019;393(10166):40-50. https://doi. org/10.1016/S0140-6736(18)32779-X.

15. Siano M, Espeli V, Mach N, et al. Gene signatures and expression of miRNAs associated with efficacy of panitumumab in a head and neck cancer phase II trial. Oral Oncol. 2018;82(May):14451. https://doi.org/10.1016/j.oraloncology.2018.05.013.

16. Fabbrocini G, Panariello L, Caro G, Cacciapuoti S. Acneiform rash induced by EGFR inhibitors: review of the literature and new insights. Ski Appendage Disord. 2015;1(1):31-7. https:// doi.org/10.1159/000371821. 
17. Mittmann N, Seung SJ. Rash rates with EGFR inhibitors: metaanalysis. Curr Oncol. 2011;18(2):e54. https://doi.org/10.3747/ co.v18i2.605.

18. Kubo A, Hashimoto H, Takahashi N, Yamada Y. Biomarkers of skin toxicity induced by anti-epidermal growth factor receptor antibody treatment in colorectal cancer. World J Gastroenterol. 2016;22(2):887-94. https://doi.org/10.3748/wjg.v22.i2.887.

19. Takahashi N, Yamada Y, Furuta K, et al. Association between serum ligands and the skin toxicity of anti-epidermal growth factor receptor antibody in metastatic colorectal cancer. Cancer Sci. 2015;106(5):604-10. https://doi.org/10.1111/cas.12642.

20. Burtness B, Goldwasser MA, Flood W, Mattar B, Forastiere AA. Phase III randomized trial of cisplatin plus placebo compared with cisplatin plus cetuximab in metastatic/recurrent head and neck cancer: an eastern cooperative oncology group study. J Clin Oncol. 2005;23(34):8646-54. https://doi.org/10.1200/ JCO.2005.02.4646.

21. Moher D, Shamseer L, Clarke M, et al. Preferred reporting items for systematic review and meta-analysis protocols (PRISMA-P) 2015 statement. Rev Esp Nutr Humana y Diet. 2016;20(2):14860. https://doi.org/10.1186/2046-4053-4-1.

22. Goldstein NI, Prewett M, Zuklys K, Rockwell P, Mendelsohn J. Biological efficacy of a chimeric antibody to the epidermal growth factor receptor in a human tumor xenograft model. Clin Cancer Res. 1995;1(11):1311-8.

23. Ciardiello F, Tortora G. EGFR antagonists in cancer treatment. N Engl J Med. 2008;358(11):1160-74. https://doi.org/10.1056/ NEJMra0707704.

24. Kimura H, Sakai K, Arao T, Shimoyama T, Tamura T, Nishio K. Antibody-dependent cellular cytotoxicity of cetuximab against tumor cells with wild-type or mutant epidermal growth factor receptor. Cancer Sci. 2007;98(8):1275-80. https://doi.org/10.1 111/j.1349-7006.2007.00510.x.

25. Patel D, Guo X, Ng S, et al. IgG isotype, glycosylation, and EGFR expression determine the induction of antibody-dependent cellular cytotoxicity in vitro by cetuximab. Hum Antibodies. 2010;19(4):89-99. https://doi.org/10.3233/HAB-2010-0232.

26. Koi L, Löck S, Linge A, et al. EGFR-amplification plus gene expression profiling predicts response to combined radiotherapy with EGFR-inhibition: a preclinical trial in 10 HNSCC-tumourxenograft models. Radiother Oncol. 2017;124(3):496-503.

27. Yamatodani T, Ekblad L, Kjellén E, Johnsson A, Mineta H, Wennerberg J. Epidermal growth factor receptor status and persistent activation of Akt and p44/42 MAPK pathways correlate with the effect of cetuximab in head and neck and colon cancer cell lines. J Cancer Res Clin Oncol. 2009;135(3):395.

28. Erjala K, Sundvall M, Junttila TT, et al. Signaling via ErbB2 and ErbB3 associates with resistance and epidermal growth factor receptor (EGFR) amplification with sensitivity to EGFR inhibitor gefitinib in head and neck squamous cell carcinoma cells. Clin Cancer Res. 2006;12(13):4103-11. https://doi.org/10.1158/10780432.CCR-05-2404.

29. Stein AP, Swick AD, Smith MA, et al. Xenograft assessment of predictive biomarkers for standard head and neck cancer therapies. Cancer Med. 2015;4(5):699-712.

30. Barnea I, Haif S, Keshet R, et al. Targeting ErbB-1 and ErbB-4 in irradiated head and neck cancer: results of in vitro and in vivo studies. Head Neck. 2013;35(3):399-407.

31. Kriegs M, Clauditz TS, Hoffer K, et al. Analyzing expression and phosphorylation of the EGF receptor in HNSCC. Sci Rep. 2019;9(1):1-8. https://doi.org/10.1038/s41598-019-49885-5.

32. Fountzilas G, Kalogera-Fountzila A, Lambaki S, et al. MMP9 but Not EGFR, MET, ERCC1, P16, and P-53 is associated with response to concomitant radiotherapy, cetuximab, and weekly cisplatin in patients with locally advanced head and neck cancer. J Oncol. 2009. https://doi.org/10.1155/2009/305908.
33. Licitra L, Mesia R, Rivera F, et al. Evaluation of EGFR gene copy number as a predictive biomarker for the efficacy of cetuximab in combination with chemotherapy in the first-line treatment of recurrent and/or metastatic squamous cell carcinoma of the head and neck: EXTREME study. Ann Oncol. 2011;22(5):107887. https://doi.org/10.1093/annonc/mdq588.

34. Licitra L, Störkel S, Kerr KM, et al. Predictive value of epidermal growth factor receptor expression for first-line chemotherapy plus cetuximab in patients with head and neck and colorectal cancer: analysis of data from the EXTREME and CRYSTAL studies. Eur J Cancer. 2013;49(6):1161-8.

35. Tinhofer I, Klinghammer K, Weichert W, et al. Expression of amphiregulin and EGFRvIII affect outcome of patients with squamous cell carcinoma of the head and neck receiving cetuximabdocetaxel treatment. Clin Cancer Res. 2011;17(15):5197-204.

36. da Costa AABA, D'Almeida Costa F, Ribeiro AR, et al. Low PTEN expression is associated with worse overall survival in head and neck squamous cell carcinoma patients treated with chemotherapy and cetuximab. Int J Clin Oncol. 2015;20(2):2829. https://doi.org/10.1007/s10147-014-0707-1.

37. Leblanc O, Vacher S, Lecerf C, et al. Biomarkers of cetuximab resistance in patients with head and neck squamous cell carcinoma. Cancer Biol Med. 2020;17(1):208-17. https://doi. org/10.20892/j.issn.2095-3941.2019.0153.

38. Kondo N, Tsukuda M, Sakakibara A, et al. Combined molecular targeted drug therapy for EGFR and HER-2 in head and neck squamous cell carcinoma cell lines. Int $\mathrm{J}$ Oncol. 2012;40(6):1805-12.

39. Jedlinski A, Ansell A, Johansson AC, Roberg K. EGFR status and EGFR ligand expression influence the treatment response of head and neck cancer cell lines. J Oral Pathol Med. 2013;42(1):26-36. https://doi.org/10.1111/j.1600-0714.2012.01177.x.

40. Stegeman H, Kaanders JH, Van Der Kogel AJ, et al. Predictive value of hypoxia, proliferation and tyrosine kinase receptors for EGFR-inhibition and radiotherapy sensitivity in head and neck cancer models. Radiother Oncol. 2013;106(3):383-9. https://doi. org/10.1016/j.radonc.2013.02.001.

41. Driehuis E, Kolders S, Spelier S, et al. Oral mucosal organoids as a potential platform for personalized cancer therapy. Cancer Discov. 2019.

42. Wheeler DL, Huang S, Kruser TJ, et al. Mechanisms of acquired resistance to cetuximab: role of HER (ErbB) family members. Oncogene. 2008;27(28):3944-56. https://doi.org/10.1038/ onc.2008.19.

43. Oliveras-Ferraros C, Cufí S, Queralt B, et al. Cross-suppression of EGFR ligands amphiregulin and epiregulin and de-repression of FGFR3 signalling contribute to cetuximab resistance in wildtype KRAS tumour cells. Br J Cancer. 2012;106(8):1406-14. https://doi.org/10.1038/bjc.2012.103.

44. Jerhammar F, Johansson A-C, Ceder R, et al. YAP1 is a potential biomarker for cetuximab resistance in head and neck cancer. Oral Oncol. 2014;50(9):832-9.

45. Ansell A, Jedlinski A, Johansson A, Roberg K. Epidermal growth factor is a potential biomarker for poor cetuximab response in tongue cancer cells. J Oral Pathol Med. 2016;45(1):9-16.

46. Kogashiwa Y, Inoue H, Kuba K, et al. Prognostic role of epiregulin/amphiregulin expression in recurrent/metastatic head and neck cancer treated with cetuximab. Head Neck. 2018;40(11):2424-31. https://doi.org/10.1002/hed.25353.

47. Hatakeyama $\mathrm{H}$, Cheng $\mathrm{H}$, Wirth $\mathrm{P}$, et al. Regulation of heparin-binding EGF-like growth factor by miR-212 and acquired cetuximab-resistance in head and neck squamous cell carcinoma. PLoS ONE. 2010;5(9):e12702.

48. Boeckx C, Blockx L, de Beeck KO, et al. Establishment and characterization of cetuximab resistant head and neck squamous 
cell carcinoma cell lines: focus on the contribution of the AP-1 transcription factor. Am J Cancer Res. 2015;5(6):1921.

49. Guarino M. Epithelial-mesenchymal transition and tumour invasion. Int J Biochem Cell Biol. 2007;39(12):2153-60. https://doi. org/10.1016/j.biocel.2007.07.011.

50. Klinghammer K, Otto R, Raguse JD, et al. Basal subtype is predictive for response to cetuximab treatment in patient-derived xenografts of squamous cell head and neck cancer. Int J Cancer. 2017;141(6):1215-21. https://doi.org/10.1002/ijc.30808.

51. Keck MK, Zuo Z, Khattri A, et al. Integrative analysis of head and neck cancer identifies two biologically distinct HPV and three non-HPV subtypes. Clin Cancer Res. 2015;21(4):870-81. https://doi.org/10.1158/1078-0432.CCR-14-2481.

52. Bossi P, Bergamini C, Siano M, et al. Functional genomics uncover the biology behind the responsiveness of head and neck squamous cell cancer patients to cetuximab. Clin Cancer Res. 2016;22(15):3961-70.

53. Zuo JH, Zhu W, Li MY, et al. Activation of EGFR promotes squamous carcinoma SCC10A cell migration and invasion via inducing EMT-like phenotype change and MMP-9-mediated degradation of E-cadherin. J Cell Biochem. 2011;112(9):2508-17. https://doi.org/10.1002/jcb.23175.

54. Kamel HFM, Al-Amodi HSAB. Exploitation of gene expression and cancer biomarkers in paving the path to era of personalized medicine. Genom Proteom Bioinform. 2017;15(4):220-35. https ://doi.org/10.1016/j.gpb.2016.11.005.

55. Tian S, Roepman P, van't Veer LJ, Bernards R, de Snoo F, Glas AM. Biological functions of the genes in the mammaprint breast cancer profile reflect the hallmarks of cancer. Biomark Insights. 2010;2010(5):129-38. https://doi.org/10.4137/BMI.S6184.

56. De Cecco L, Giannoccaro M, Marchesi E, et al. Integrative miRNA-gene expression analysis enables refinement of associated biology and prediction of response to cetuximab in head and neck squamous cell cancer. Genes (Basel). 2017;8(1):35.

57. Braig F, Voigtlaender M, Schieferdecker A, et al. Liquid biopsy monitoring uncovers acquired RAS-mediated resistance to cetuximab in a substantial proportion of patients with head and neck squamous cell carcinoma. Oncotarget. 2016;7(28):42988.

58. Braig F, Kriegs M, Voigtlaender M, et al. Cetuximab resistance in head and neck cancer is mediated by EGFR-K521 polymorphism. Cancer Res. 2017;77(5):1188-99.

59. da Costa AABA, Costa FD, Araújo DV, et al. The roles of PTEN, cMET, and p16 in resistance to cetuximab in head and neck squamous cell carcinoma. Med Oncol. 2019;36(1):1-9. https://doi. org/10.1007/s12032-018-1234-0.

60. Kondo T, Ozawa S, Ikoma T, et al. Expression of the chemokine CXCL14 and cetuximab-dependent tumour suppression in head and neck squamous cell carcinoma. Oncogenesis. 2016;5(7):e240.

61. Brand TM, Iida M, Stein AP, et al. AXL mediates resistance to cetuximab therapy. Cancer Res. 2014;74(18):5152-64. https:// doi.org/10.1158/0008-5472.CAN-14-0294.

62. Rebucci M, Peixoto P, Dewitte A, et al. Mechanisms underlying resistance to cetuximab in the HNSCC cell line: role of AKT inhibition in bypassing this resistance. Int $\mathrm{J}$ Oncol. 2011;38(1):189-200.

63. Weidhaas JB, Harris J, Schaue D, et al. The KRAS-variant and cetuximab response in head and neck squamous cell cancer a secondary analysis of a randomized clinical trial. JAMA Oncol. 2017;3(4):483-91. https://doi.org/10.1001/jamaoncol.2016.5478.

64. Eze N, Lee JW, Yang DH, et al. PTEN loss is associated with resistance to cetuximab in patients with head and neck squamous cell carcinoma. Oral Oncol. 2019;91(February):69-78. https:// doi.org/10.1016/j.oraloncology.2019.02.026.

65. Izumi $\mathrm{H}$, Wang Z, Goto $\mathrm{Y}$, et al. Pathway-specific genome editing of PI3K/mTOR tumor suppressor genes reveals that PTEN loss contributes to cetuximab resistance in head and neck cancer. Mol Cancer Ther. 2020;19(7):1562-71. https://doi.org/10.1158/15357163.MCT-19-1036.

66. Mriouah J, Boura C, Pinel S, et al. Cellular response to cetuximab in PTEN-silenced head and neck squamous cell carcinoma cell line. Int J Oncol. 2010;37(6):1555-63.

67. Argiris A, Lee SC, Feinstein T, et al. Serum biomarkers as potential predictors of antitumor activity of cetuximab-containing therapy for locally advanced head and neck cancer. Oral Oncol. 2011;47(10):961-6.

68. Espinosa-Cotton M, Fertig EJ, Stabile LP, et al. A preliminary analysis of interleukin-1 ligands as potential predictive biomarkers of response to cetuximab. Biomark Res. 2019;7(1):1-11. https://doi.org/10.1186/s40364-019-0164-0.

69. Lyu J, Song H, Tian Z, Miao Y, Ren G, Guo W. Predictive value of pAKT/PTEN expression in oral squamous cell carcinoma treated with cetuximab-based chemotherapy. Oral Surg Oral Med Oral Pathol Oral Radiol. 2016;121(1):67-72.

70. Bossi P, Siano M, Bergamini C, et al. Are Fusion Transcripts in Relapsed/Metastatic Head and Neck Cancer Patients Predictive of Response to Anti-EGFR Therapies? Dis Markers. 2017;2017.

71. Kiaris H, Spandidos DA, Jones AS, Vaughan ED, Field JK. Mutations, expression and genomic instability of the H-ras protooncogene in squamous cell carcinomas of the head and neck. Br J Cancer. 1995;72(1):123-8. https://doi.org/10.1038/bjc.1995.287.

72. Lea IA, Jackson MA, Li X, Bailey S, Peddada SD, Dunnick JK. Genetic pathways and mutation profiles of human cancers: site- and exposure-specific patterns. Carcinogenesis. 2007;28(9):1851-8. https://doi.org/10.1093/carcin/bgm176.

73. Vermorken JB, Stöhlmacher-Williams J, Davidenko I, et al. Cisplatin and fluorouracil with or without panitumumab in patients with recurrent or metastatic squamous-cell carcinoma of the head and neck (SPECTRUM): an open-label phase 3 randomised trial. Lancet Oncol. 2013;14(8):697-710. https://doi.org/10.1016/ S1470-2045(13)70181-5.

74. Lurje G, Lenz HJ. EGFR signaling and drug discovery. Oncology. 2010;77(6):400-10. https://doi.org/10.1159/000279388.

75. Hickinson DM, Marshall GB, Beran GJ, et al. Identification of biomarkers in human head and neck tumor cell lines that predict for in vitro sensitivity to gefitinib. Clin Transl Sci. 2009;2(3):18392. https://doi.org/10.1111/j.1752-8062.2009.00099.x.

76. Rogers SJ, Box C, Chambers P, et al. Determinants of response to epidermal growth factor receptor tyrosine kinase inhibition in squamous cell carcinoma of the head and neck. J Pathol A J Pathol Soc Gt Britain Irel. 2009;218(1):122-30.

77. Nichols AC, Black M, Yoo J, et al. Exploiting high-throughput cell line drug screening studies to identify candidate therapeutic agents in head and neck cancer. BMC Pharmacol Toxicol. 2014; 15(1):66.

78. Tepper SR, Zuo Z, Khattri A, Heß J, Seiwert TY. Growth factor expression mediates resistance to EGFR inhibitors in head and neck squamous cell carcinomas. Oral Oncol. 2016;56:62-70.

79. Tan EH, Goh C, Lim WT, et al. Gefitinib, cisplatin, and concurrent radiotherapy for locally advanced head and neck cancer: EGFR FISH, protein expression, and mutational status are not predictive biomarkers. Ann Oncol. 2012;23(4):1010-6. https:// doi.org/10.1093/annonc/mdr327.

80. Cohen EEW, Lingen MW, Martin LE, et al. Response of some head and neck cancers to epidermal growth factor receptor tyrosine kinase inhibitors may be linked to mutation of ERBB2 rather than EGFR. Clin Cancer Res. 2005;11(22):8105-8. https://doi. org/10.1158/1078-0432.CCR-05-0926.

81. Bill A, Gutierrez A, Kulkarni S, et al. ANO1/TMEM16A interacts with EGFR and correlates with sensitivity to EGFR-targeting therapy in head and neck cancer. Oncotarget. 2015;6(11):9173. 
82. Weisheit S, Liebmann C. Allosteric modulation by protein kinase $\mathrm{C} \varepsilon$ leads to modified responses of EGF receptor towards tyrosine kinase inhibitors. Cell Signal. 2012;24(2):422-34. https://doi. org/10.1016/j.cellsig.2011.09.010.

83. Thariat J, Bensadoun RJ, Etienne-Grimaldi MC, et al. Contrasted outcomes to gefitinib on tumoral IGF1R expression in head and neck cancer patients receiving postoperative chemoradiation (GORTEC trial 2004-02). Clin Cancer Res. 2012;18(18):512333. https://doi.org/10.1158/1078-0432.CCR-12-1518.

84. Frederick BA, Helfrich BA, Coldren CD, et al. Epithelial to mesenchymal transition predicts gefitinib resistance in cell lines of head and neck squamous cell carcinoma and non-small cell lung carcinoma. Mol Cancer Ther. 2007;6(6):1683-91.

85. Timpson P, Wilson AS, Lehrbach GM, Sutherland RL, Musgrove EA, Daly RJ. Aberrant expression of cortactin in head and neck squamous cell carcinoma cells is associated with enhanced cell proliferation and resistance to the epidermal growth factor receptor inhibitor gefitinib. Cancer Res. 2007;67(19):9304-14. https ://doi.org/10.1158/0008-5472.CAN-07-0798.

86. Box C, Mendiola M, Gowan S, et al. A novel serum protein signature associated with resistance to epidermal growth factor receptor tyrosine kinase inhibitors in head and neck squamous cell carcinoma. Eur J Cancer. 2013;49(11):2512-21.

87. Tan DSW, Chong FT, Leong HS, et al. Long noncoding RNA EGFR-AS1 mediates epidermal growth factor receptor addiction and modulates treatment response in squamous cell carcinoma. Nat Med. 2017;23(10):1167.

88. Ngan HL, Poon PHY, Su YX, et al. Erlotinib sensitivity of MAPK1p.D321N mutation in head and neck squamous cell carcinoma. npj Genomic Med. 2020;5(1):1-5. https://doi. org/10.1038/s41525-020-0124-5.

89. Van Allen EM, Lui VWY, Egloff AM, et al. Genomic correlate of exceptional erlotinib response in head and neck squamous cell carcinoma. JAMA Oncol. 2015;1(2):238-44. https://doi. org/10.1001/jamaoncol.2015.34.

90. Liu S, Wang Y, Han Y, et al. EREG-driven oncogenesis of head and neck squamous cell carcinoma exhibits higher sensitivity to Erlotinib therapy. Theranostics. 2020;10(23):10589-605. https ://doi.org/10.7150/thno.47176.

91. Dennis M, Wang G, Luo J, et al. Snail controls the mesenchymal phenotype and drives erlotinib resistance in Oral epithelial and HNSCC cells. Otolaryngol Head Neck Surg. 2014;147(4):72632. https://doi.org/10.1177/0194599812446407.Snail.

92. Muller S, Su L, Tighiouart M, et al. Distinctive E-cadherin and epidermal growth factor receptor expression in metastatic and nonmetastatic head and neck squamous cell carcinoma: predictive and prognostic correlation. Cancer. 2008;113(1):97-107. https://doi.org/10.1002/cncr.23557.

93. Haddad Y, Choi W. The transcriptional repressor Delta EF1 controls the EMT phenotype and resistance to the EGFR inhibitor erlotinib in human head \& neck squamous cell. Clin Cancer Res. 2009;15(2):532-42. https://doi.org/10.1158/1078-0432.CCR-081733.The.

94. Chan CY, Chang CM, Chen YH, Sheu JJC, Lin TY, Huang CY. Regulatory role of transcription factor HBP1 in anticancer efficacy of EGFR inhibitor erlotinib in HNSCC. Head Neck. 2020;42(10):2958-67. https://doi.org/10.1002/hed.26346.

95. Chau NG, Perez-Ordonez B, Zhang K, et al. The association between EGFR variant III, HPV, p16, c-MET, EGFR gene copy number and response to EGFR inhibitors in patients with recurrent or metastatic squamous cell carcinoma of the head and neck. Head Neck Oncol. 2011;3(1):11.

96. Thomas F, Delmar P, Vergez S, et al. Gene expression profiling on pre-and post-erlotinib tumors from patients with head and neck squamous cell carcinoma. Head Neck. 2013;35(6):809-18.
97. Machiels JP, Bossi P, Menis J, et al. Activity and safety of afatinib in a window preoperative EORTC study in patients with squamous cell carcinoma of the head and neck (SCCHN). Ann Oncol. 2018;29(4):985-91. https://doi.org/10.1093/annon $\mathrm{c} / \mathrm{mdy} 013$.

98. Silva-Oliveira RJ, Melendez M, Martinho O, et al. AKT can modulate the in vitro response of HNSCC cells to irreversible EGFR inhibitors. Oncotarget. 2017;8(32):53288.

99. Young NR, Soneru C, Liu J, et al. Afatinib efficacy against squamous cell carcinoma of the head and neck cell lines in vitro and in vivo. Target Oncol. 2015;10(4):501-8.

100. Cohen EEW, Licitra LF, Burtness B, et al. Biomarkers predict enhanced clinical outcomes with afatinib versus methotrexate in patients with second-line recurrent and/or metastatic head and neck cancer. Ann Oncol. 2017;28(10):2526-32. https://doi. org/10.1093/annonc/mdx344.

101. Greshock J, Cheng J, Rusnak D, et al. Genome-wide DNA copy number predictors of lapatinib sensitivity in tumor-derived cell lines. Mol Cancer Ther. 2008;7(4):935-43.

102. Beizaei K, Gleißner L, Hoffer K, et al. Receptor tyrosine kinase MET as potential target of multi-kinase inhibitor and radiosensitizer sorafenib in HNSCC. Head Neck. 2019;41(1):208-15.

103. Huang KK, Jang KW, Kim S, et al. Exome sequencing reveals recurrent REV3L mutations in cisplatin-resistant squamous cell carcinoma of head and neck. Sci Rep. 2016;6:19552.

104. Silva-Oliveira RJ, Silva VAO, Martinho O, et al. Cytotoxicity of allitinib, an irreversible anti-EGFR agent, in a large panel of human cancer-derived cell lines: KRAS mutation status as a predictive biomarker. Cell Oncol. 2016;39(3):253-63.

105. Ruicci KM, Meens J, Sun RX, et al. A controlled trial of HNSCC patient-derived xenografts reveals broad efficacy of PI3K $\alpha$ inhibition in controlling tumor growth. Int J Cancer. 2018. https:// doi.org/10.1002/ijc.32009.

106. Mazumdar T, Byers LA, Ng PKS, et al. A comprehensive evaluation of biomarkers predictive of response to PI3K inhibitors and of resistance mechanisms in head and neck squamous cell carcinoma. Mol Cancer Ther. 2014;13(11):2738-50. https://doi. org/10.1158/1535-7163.MCT-13-1090.

107. Sambandam V, Frederick MJ, Shen L, et al. PDK1 mediates Notch1-mutated head and neck squamous carcinoma vulnerability to therapeutic PI3K/mTOR inhibition. Clin Cancer Res. 2019;25(11):3329-40. https://doi.org/10.1158/1078-0432. CCR-18-3276.

108. Klinghammer K, Politz O, Eder T, et al. Combination of copanlisib with cetuximab improves tumor response in cetuximabresistant patient-derived xenografts of head and neck cancer. Oncotarget. 2020;11(41):3688-97. https://doi.org/10.18632/ oncotarget. 27763.

109. Soulieres D, Licitra L, Mesía R, et al. Molecular alterations and buparlisib efficacy in patients with squamous cell carcinoma of the head and neck: biomarker analysis from BERIL-1. Clin Cancer Res. 2018;24(11):2505-16. https://doi.org/10.1158/10780432.CCR-17-2644.

110. Yamaguchi K, Iglesias-Bartolomé R, Wang Z, et al. A syntheticlethality RNAi screen reveals an ERK-mTOR co-targeting pro-apoptotic switch in PIK3CA+ oral cancers. Oncotarget. 2016;7(10):10696.

111. Niehr F, Weichert W, Stenzinger A, Budach V, Tinhofer I. CCI779 (Temsirolimus) exhibits increased anti-tumor activity in low EGFR expressing HNSCC cell lines and is effective in cells with acquired resistance to cisplatin or cetuximab. J Transl Med. 2015;13(1): 106

112. Klinghammer K, Raguse JD, Plath T, et al. A comprehensively characterized large panel of head and neck cancer patient-derived xenografts identifies the mTOR inhibitor everolimus as potential 
new treatment option. Int J Cancer. 2015;136(12):2940-8. https ://doi.org/10.1002/ijc.29344.

113. John K, Rösner I, Keilholz U, Gauler T, Bantel H, Grünwald V. Baseline caspase activity predicts progression free survival of temsirolimus-treated head neck cancer patients. Eur J Cancer. 2015;51(12):1596-602.

114. Grünwald V, Keilholz U, Boehm A, et al. TEMHEAD: a singlearm multicentre phase II study of temsirolimus in platin- and cetuximab refractory recurrent and/or metastatic squamous cell carcinoma of the head and neck (SCCHN) of the German SCCHN Group (AIO). Ann Oncol. 2015;26(3):561-7. https:// doi.org/10.1093/annonc/mdu571.

115. Viloria-Petit A, Kerbel RS, Jothy S, et al. Acquired resistance to the antitumor effect of epidermal growth factor receptor-blocking antibodies in vivo: a role for altered tumor angiogenesis. Cancer Res. 2001;61(13):5090-101.

116. Brand TM, Iida M, Wheeler DL. Molecular mechanisms of resistance to the EGFR monoclonal antibody cetuximab. Cancer Biol Ther. 2011;11(9):777-92. https://doi.org/10.4161/ cbt.11.9.15050.

117. Huang L, Fu L. Mechanisms of resistance to EGFR tyrosine kinase inhibitors. Acta Pharm Sin B. 2015;5(5):390-401. https ://doi.org/10.1016/j.apsb.2015.07.001.

118. Economopoulou P, Agelaki S, Perisanidis C, Giotakis EI, Psyrri A. The promise of immunotherapy in head and neck squamous cell carcinoma. Ann Oncol. 2016;27(9):1675-85. https://doi. org/10.1093/annonc/mdw226.

119. Biankin AV, Piantadosi S, Hollingsworth SJ. Patient-centric trials for therapeutic development in precision oncology. Nature. 2015;526(7573):361-70. https://doi.org/10.1038/nature15819.

120. Roepman P, Wessels LFA, Kettelarij N, et al. An expression profile for diagnosis of lymph node metastases from primary head and neck squamous cell carcinomas. Nat Genet. 2005;37(2):1826. https://doi.org/10.1038/ng1502.

121. Gao D, Vela I, Sboner A, et al. Organoid cultures derived from patients with advanced prostate cancer. Cell. 2014;159(1):17687. https://doi.org/10.1016/j.cell.2014.08.016.

122. Van De Wetering M, Francies HE, Francis JM, et al. Prospective derivation of a living organoid biobank of colorectal cancer patients. Cell. 2015;161(4):933-45. https://doi.org/10.1016/j. cell.2015.03.053.

123. Fujii M, Shimokawa M, Date S, et al. A colorectal tumor organoid library demonstrates progressive loss of niche factor requirements during tumorigenesis. Cell Stem Cell. 2016;18(6):827-38. https://doi.org/10.1016/j.stem.2016.04.003.

124. Vlachogiannis G, Hedayat S, Vatsiou A, et al. Patient-derived organoids model treatment response of metastatic gastrointestinal cancers. Science (80-). 2018;359(6378):920-6. https://doi. org/10.1126/science.aao2774. 